\title{
Prácticas de responsabilidad sostenible de cadenas de suministro: Revisión y propuesta
}

\author{
Cogollo Flórez, Juan Miguel ${ }^{1}$ \\ Ruiz Vásquez, Camilo²
}

\section{Resumen}

El desarrollo sostenible de las organizaciones y sus cadenas de suministro es un aspecto fundamental y relevante en los negocios modernos y se basa en la conservación del medio ambiente, la satisfacción de necesidades sociales y el logro del progreso económico. Por ello, en este artículo se hace un estudio del concepto de Responsabilidad Sostenible de Cadenas de Suministro, identificando enfoques teóricos y prácticas propuestas en la literatura, a través de una revisión sistemática de literatura en bases de datos científicas en el periodo comprendido entre los años 1997 y 2018. Se identificaron los principales aportes y aspectos de las prácticas para un desempeño sostenible de las cadenas de suministro en las dimensiones económica, social y ambiental. Finalmente, con base en los referentes teóricos consultados, se propone una metodología general para la implementación de prácticas de responsabilidad sostenible en cadenas de suministro.

Palabras clave: desarrollo sostenible; responsabilidad sostenible; cadena de suministro; prácticas.

Recibido: 25.11.18 Aceptado: 15.05.19

1 Profesor Asociado, Departamento de Calidad y Producción, Instituto Tecnológico Metropolitano - ITM, Medellín, Colombia. E-mail: juancogollo@itm.edu.co, ORCID: 0000-0002-6101-3134

2 Investigador, Departamento de Calidad y Producción, Instituto Tecnológico Metropolitano ITM, Medellín, Colombia. E-mail: camiloruiz188751@correo.itm.edu.co. 


\title{
Supply chains sustainable responsibility practices: review and proposal
}

\begin{abstract}
Sustainable development of organizations and supply chains is a fundamental and relevant aspect in modern business; it is based on the environment conservation, social needs satisfaction and achievement of economic progress. Therefore, this paper studies the concept of Supply Chains Sustainable Responsibility (SCSR), by identifying theoretical approaches and practices proposed in the literature, through a systematic literature review in scientific databases, in the period between 1997 and 2018. The main contributions and aspects regarding on the practices aimed to supply chains sustainable performance were identified, this is, the economic, social and environmental dimensions. Finally, based on the consulted theoretical references, we proposed a general methodology for implementing supply chains sustainable responsibility practices.
\end{abstract}

Keywords: Sustainable development; sustainable responsibility; supply chain; practices.

\section{Introducción}

En el contexto empresarial moderno de operaciones globales, las organizaciones están cambiando el enfoque de actuación frente a las problemáticas sociales de su entorno directo e indirecto. Es así como en el diseño y gestión de las cadenas de suministro se ha incorporado aspectos de responsabilidad social y desempeño sostenible que influyen en el flujo de los productos y servicios desde los proveedores hasta el cliente final. Este enfoque de cadenas de suministro responsables y sostenibles es de gran utilidad y es producto de la integración y evolución de la Responsabilidad Social Empresarial (RSE), el enfoque del Triple Balance de la sostenibilidad (económico, social y ambiental) y la Gestión de Cadena de Suministro, para así priorizar los retos modernos del mejoramiento a través del concepto de Responsabilidad Sostenible de Cadenas de Suministro (RSCS).

Una cadena de suministro está integrada por las organizaciones que tienen contacto con un producto en su flujo desde los proveedores hasta el cliente final e incluye las empresas encargadas de ensamblar y entregar, es decir, que está compuesta por todas las partes interesadas $\mathrm{y}$, por ende, por las complejidades que se generan para la coordinación y colaboración mutua (Ferrara et al, 2017:1) we establish a dynamic game to allocate CSR (Corporate Social Responsibility. La globalización de mercados ha originado el consecuente incremento de la complejidad de la gestión en las cadenas de suministro. Esta problemática y el concepto de desarrollo sostenible se ha manifestado a medida que las personas son cada vez más 
conscientes del impacto que generan sus comportamientos de consumo a nivel global, lo cual conlleva a que se requiera un grado de compromiso por parte de las organizaciones y sus cadenas de suministro con la finalidad implementar mejoras en los diferentes ámbitos de la sostenibilidad (Hutchins y Sutherland, 2008:1693).

El ámbito sostenible es un factor clave para la ejecución de mejoras en las organizaciones por medio del enfoque del Triple Balance (económico, social y ambiental), para efectuar cambios con el fin de aumentar el impacto del desarrollo sostenible a nivel global. En consiguiente, una organización socialmente responsable debe estimar que el impacto de sus productos tenga una visión más amplia al área operacional y sus respectivos ciclos de vida, también llamado como cadena de suministro responsable o cadena de suministro extendida (Benoit et al, 2012:1947). Por el contrario, las organizaciones que sigan realizando operaciones estratégicas en la parte económica como fuente de interés principal, dejando a un lado el ámbito social y el ambiental, estarían generando algunas problemáticas por estos factores que son críticos en relación al desarrollo sostenible. También, las organizaciones que interactúen de forma independiente y no vinculadas a su cadena de suministro, frecuentemente se ven afectadas por comportamientos no adecuados de otras organizaciones (Wang, 2013:2985).

No obstante, desde el ámbito de la gestión de la calidad se han desarrollado distintas normas con base en los aspectos social, económico y ambiental (ISO 14000, ISO 26000, ISO 20400 , ISO 45000 , entre otras), cuyas certificaciones son voluntarias y son adoptadas por aquellas organizaciones que desean obtener mejores beneficios en procesos contractuales o como requisitos puntuales.

Este artículo es resultado de un proyecto de investigación que tiene como objetivo principal identificar los diferentes enfoques y metodologías que se han propuesto en la literatura para el estudio del concepto de Responsabilidad Sostenible de la Cadena de Suministro (RSCS).

La investigación inicia con un estudio del concepto de Responsabilidad Sostenible de Cadenas de Suministro, realizando una evolución e integración de los conceptos de Responsabilidad Empresarial y el enfoque del Triple Balance de la sostenibilidad (económico, social y ambiental) en el contexto de las cadenas de suministro. La revisión de literatura sobre aportes teóricos y prácticos realizados entre los años 1997 y 2018 es esencial para el análisis de modelos y casos de estudio de RSCS, evidenciando la importancia creciente del tema en los ámbitos académico e industrial.

La metodología de revisión sistemática de literatura asumida, permitirá identificar los principales aportes teóricos y de prácticas de RSCS; contribución importante en el estudio de este constructo emergente en la gestión moderna de cadenas de suministro.

\section{Aportes teóricos y prácticas de Responsabilidad Sostenible de la Cadena de Suministro}

Para identificar las contribuciones más importantes que se han realizado del año 1997 al 2018 sobre las prácticas de Responsabilidad Sostenible de Cadenas 
de Suministro (RSCS), se aplicó la metodología de Revisión Sistemática de Literatura propuesta por Kitchenham (2004), la cual facilitó la búsqueda, análisis y síntesis de la evidencia documental en el periodo de tiempo establecido, como punto de partida para la elaboración de la metodología de implementación de prácticas de RSCS. En las siguientes subsecciones se desarrollan cada uno de los pasos de la metodología (diagrama 1).

\section{Diagrama 1 \\ Etapas de la metodología empleada en la investigación}

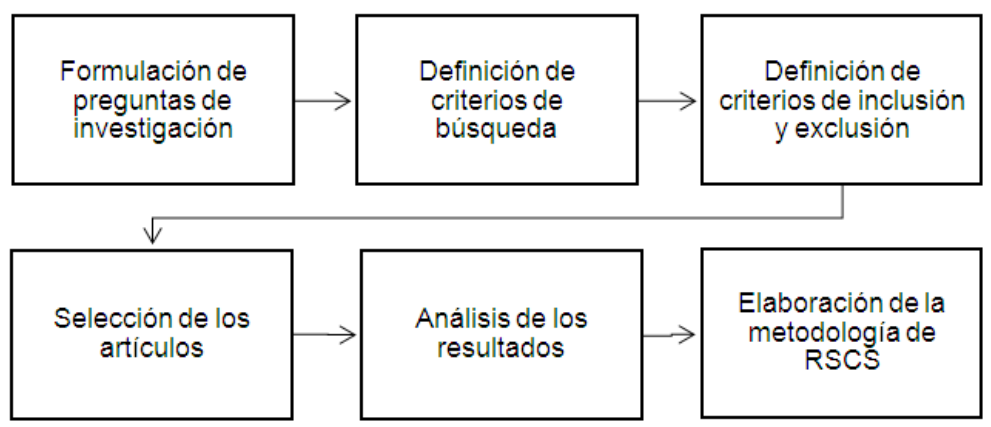

Fuente: Kitchenham (2004)

En este orden de ideas, la formulación de preguntas de investigación es el punto de inicio que conduce el progreso de las subsiguientes etapas, con la finalidad de identificar cómo se ha abordado desde la literatura científica la temática de la Responsabilidad Sostenible de Cadenas de Suministro. En base a los planteamientos anteriores, se formularon las siguientes preguntas de investigación:

P1: ¿Cuáles son los planteamientos teóricos de RSCS que han sido propuestos desde el año 1997 hasta el año 2018?

P2: ¿Cuáles son los aportes sobre prácticas de RSCS que han sido propuestos desde el año 1997 hasta el año 2018?

Los lineamientos del proceso de búsqueda se diseñaron basados en el constructo de la investigación: prácticas de Responsabilidad Sostenible de Cadena de Suministro (RSCS). Así, se realizó una búsqueda manual en las bases de datos Scopus y Science Direct, de los artículos científicos publicados desde el año 1997 hasta el año 2018. Se filtraron cadenas de búsqueda contemplando los títulos y luego se definieron las palabras clave para obtener un amplio repertorio del tema, y así, abarcar ampliamente el tema de estudio. Para el proceso de búsqueda se desarrollaron las siguientes palabras clave con sus correspondientes conectores:

\footnotetext{
- "sustainable responsibility" AND "supply chain"
} 
- "sustainable responsibility" AND "supply chain management"

- "supply chain sustainable
responsibility"
"supply chain sustainable
responsibility" AND "practices"

La selección de los artículos obtenidos en el proceso de búsqueda anterior se llevó a cabo considerando dos criterios de inclusión: (i) el artículo presenta un aporte teórico sobre aspectos básicos de RSCS, y, (ii) el artículo presenta un esquema general de aplicación de prácticas de RSCS en casos reales. Análogamente, se excluyeron del estudio los artículos que cumplieron alguno de los siguientes criterios de exclusión: (i) el artículo presenta un aporte sobre responsabilidad social empresarial sin considerar el contexto de las cadenas de suministro, y (ii) el artículo presenta aporte sobre cadenas de suministro sin considerar la responsabilidad social, económica o ambiental.

En esta sección se presentan y discuten los resultados obtenidos con la aplicación de las etapas descritas anteriormente, teniendo como referente las preguntas de investigación planteadas; esto es, los aportes teóricos y prácticas de RSCS identificadas en literatura, al igual que se describe la metodología desarrollada para la implementación de prácticas de RSCS, con base en los resultados de la revisión de literatura previa.

La búsqueda inicial fue realizada usando los criterios de búsqueda descritos previamente y arrojó un total de 785 artículos. Después de aplicar los criterios de inclusión y exclusión, se seleccionaron 30 artículos definitivos, cuyos autores, año de publicación y principal aporte o temática se describen en el cuadro 1.

\section{Cuadro 1 \\ Descripción de los artículos seleccionados}

\begin{tabular}{ccl}
\hline$N^{\circ}$ & Autor (año de publicación) & \multicolumn{1}{c}{ Temática } \\
\hline 1 & Boyd et al, (2007) & $\begin{array}{l}\text { Responsabilidad social corporativa en las cadenas de suministro } \\
\text { globales: una perspectiva de justicia procesal. }\end{array}$ \\
2 & Ciliberti et al, (2008) & $\begin{array}{l}\text { Investigar la responsabilidad social corporativa en las cadenas de } \\
\text { suministro: una perspectiva de las PYMES. }\end{array}$ \\
3 & Hutchins y Sutherland (2008) & $\begin{array}{l}\text { Una exploración de las medidas de sostenibilidad social y su } \\
\text { aplicación a las decisiones de la cadena de suministro. }\end{array}$ \\
4 & Parmigiani et al, (2011) & $\begin{array}{l}\text { Relación entre eficiencia y la responsabilidad social: implicaciones } \\
\text { de rendimiento de la configuración, el control y las capacidades de } \\
\text { la cadena de suministro. } \\
\text { Identificación de los impactos sociales en las cadenas de } \\
\text { suministro de productos: descripción general y aplicación de la } \\
\text { base de datos de puntos de acceso sociales. }\end{array}$ \\
\hline
\end{tabular}




\section{Cont... Cuadro 1}

Kogg y Mont (2012)
Baumann et al, (2013)

Wang y Baoying (2013)

Fernández et al, (2014)

Quarshie et al, (2016)

Bisogno (2016)

Chinomona y Omoruyi (2016)

Ağan et al, (2016)

Mariadoss et al, (2016)

Sancha et al, (2016)

Mani et al, (2016)

Rueda et al, (2017)

Shen et al, (2017)

Feng et al, (2017)

Wei y Huang. (2017)

Gold y Schleper. (2017)

Sudarto et al, (2017)

Rahim (2017)

Pedram et al, (2017)

Ferrara et al, (2017)
Responsabilidad ambiental y social en las cadenas de suministro: la práctica de la elección y la gestión entre organizaciones.

Organización de la responsabilidad social corporativa en pequeñas y grandes empresas: el tamaño importa.

Investigación sobre la responsabilidad social corporativa del sistema de la cadena de suministro basada en la teoría de la autoorganización.

Compromiso con la responsabilidad social corporativa medido a través de informes de iniciativas de informes globales: factores que afectan el comportamiento de las empresas.

Sostenibilidad y responsabilidad social corporativa en las cadenas de suministro: el estado de la investigación en administración de cadenas de suministro y revistas de ética empresarial.

Responsabilidad social corporativa y cadenas de suministro: contribución a la sostenibilidad del bienestar.

La influencia de la RSE, la innovación y la asociación en la cadena de suministro en la competitividad de la firma.

Las relaciones entre responsabilidad social corporativa, desarrollo de proveedores ambientales y desempeño de las empresas.

Influencias de las orientaciones de las empresas en la gestión sostenible de la cadena de suministro.

Cadena de suministro socialmente responsable a través de la evaluación y la colaboración.

Sostenibilidad social de la cadena de suministro para los países en desarrollo: evidencia de India.

Inversiones corporativas en la sostenibilidad de la cadena de suministro: selección de instrumentos en la industria agroalimentaria.

Aspectos de sostenibilidad en las cadenas de suministro de textiles y prendas de vestir.

Responsabilidad social corporativa para la gestión de la cadena de suministro: revisión de la literatura y análisis bibliométrico.

El sistema de trazabilidad de alimentos como una buena práctica de responsabilidad social corporativa en el sector de restaurantes.

Un camino hacia la verdadera sostenibilidad: una base de reconocimiento para la gestión sostenible de la cadena de suministro.

Planificación eficiente y flexible a largo plazo de la capacidad para lograr dimensiones óptimas de sostenibilidad: rendimiento de la logística social inversa.

Mejora de la responsabilidad social en las industrias de a través de un nuevo enfoque de gobernanza en las leyes.

Desarrollo de la red de la cadena de suministro de circuito cerrado en términos de responsabilidad social corporativa.

Propuesta de un juego dinámico de la cadena de suministro para una responsabilidad social corporativa. 


\section{Cont... Cuadro 1}

\begin{tabular}{|c|c|c|}
\hline 26 & Yawar y Seuring (2018) & $\begin{array}{l}\text { El papel del desarrollo de proveedores en la gestión de problemas } \\
\text { sociales y sociales en las cadenas de suministro. }\end{array}$ \\
\hline 27 & Hong et al, (2018) & $\begin{array}{l}\text { Prácticas sostenibles de gestión de la cadena de suministro, } \\
\text { capacidades dinámicas de la cadena de suministro y rendimiento } \\
\text { empresarial. }\end{array}$ \\
\hline 28 & Mani et al, (2018) & $\begin{array}{l}\text { Mejora del rendimiento de la cadena de suministro a través de la } \\
\text { sostenibilidad social del proveedor: una perspectiva de economía } \\
\text { emergente. }\end{array}$ \\
\hline 29 & Yadlapalli et al, (2018) & $\begin{array}{l}\text { Mecanismos de gobierno socialmente responsables para las } \\
\text { empresas manufactureras en las cadenas de suministro de } \\
\text { prendas de vestir. }\end{array}$ \\
\hline 30 & Soler (2018) & $\begin{array}{l}\text { Revisión de modelos para evaluar la sostenibilidad de las } \\
\text { organizaciones. }\end{array}$ \\
\hline
\end{tabular}

Fuente: Elaboración propia

\section{Responsabilidad Sostenible de Cadenas de Suministro: Enfoques teóricos}

La sostenibilidad se puede explicar como la satisfacción a las necesidades de las generaciones presentes y futuras, comprendiendo la satisfacción de las necesidades entre las partes existentes como lo son empleados, clientes, entre otros (Boyd et al, 2007). Por otra parte, la sostenibilidad social es la encargada de gestionar los asuntos relacionados con la supervivencia de las organizaciones en un determinado periodo de tiempo, por medio de aplicación de prácticas sostenibles que sean llevadas a cabo entre las partes interesadas (Mani et al, 2016:43).

También, hay gran variedad en referencia a la integración de estrategias con el fin de lograr objetivos sociales, económicos y ambientales en una empresa para efectuar una coordinación acertada en procesos para la mejora del desempeño económico, a largo plazo de la empresa individualmente y sus cadenas de suministro, estos términos se adoptan como el enfoque del Triple Balance (diagrama 2) (Ağan et al, $2016: 1873)$ which is the development of suppliers to manufacturers for the purpose of environmental performance. Corporate social responsibility (CSR).

En este, se asocian diferentes aspectos para la evaluación del desempeño sostenible de las empresas en las dimensiones económica (que agrupa aspectos como costos de producción, costos logísticos, costos de calidad y no calidad, entre otros), social (que une la atención a los recursos humanos, la salud, satisfacción de empleados y clientes) y ambiental (relacionada con el cuidado de los recursos, la protección a la biodiversidad, la producción sana, desperdicios por productos defectuosos o devoluciones, entre otros) (Plascencia et al, 2018:65). 


\section{Diagrama 2 \\ Pilares del Triple Balance y algunos indicadores relacionados}

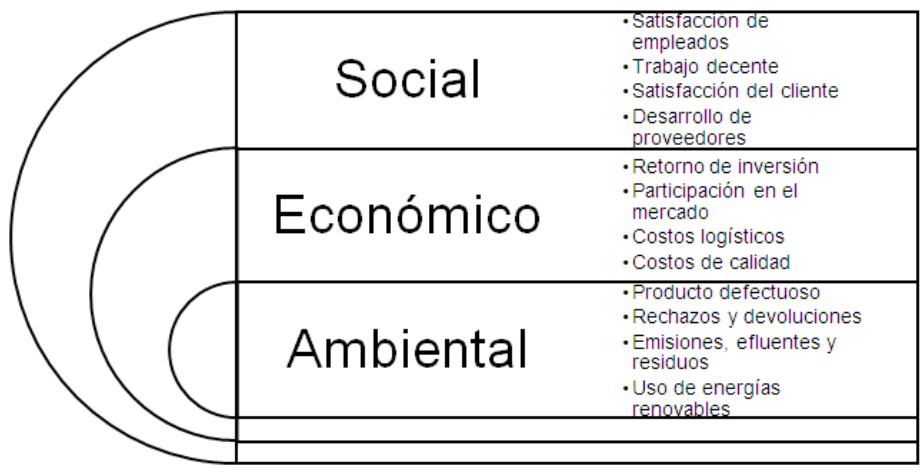

Fuente: Elaboración propia.

La responsabilidad social es un motivador para que los que interactúan en la cadena de suministro trabajen en conjunto para obtener beneficios e ingresos extras dando cumplimiento a cada estrategia utilizada (Sudarto et al, 2017:47) a single-product System Dynamics (SD). Las organizaciones deben indagar acerca de mejores maneras para obtener proveedores exitosos por medio de esfuerzos que sugieran más empleados y uso eficiente de materias primas (Ağan et al, 2016:1879).

La sostenibilidad corporativa comprende la satisfacción de las necesidades de las partes interesadas de una organización, sean agentes directos e indirectos, como clientes, comunidad, accionistas, empleados, entre otros (Gold y Schleper, 2017:426). Con respecto a las discusiones que se generan con los problemas sociales en cadenas de suministro, es necesario tener claridad sobre cómo enfrentarlos y cómo las empresas deben ser socialmente receptivas conforme a las partes interesadas, para así obtener una ventaja que sea sostenible. Otros estudios, como los de Mani et al (2016:43), consideran que también son importantes la seguridad, la equidad en las organizaciones y los derechos humanos en las prácticas sociales en las cadenas de suministro, aunado a la necesidad de un liderazgo fuerte en la cadena de suministro para promover e implementar un mayor rendimiento sostenible (Shen et al. 2017:2).

Por lo tanto, la RSCS requiere de un enfoque de gestión basado en comunicación efectiva, confianza, honestidad, transparencia y beneficios mutuos para todos los integrantes de la cadena de suministro. Esta asociación de cadena de suministro es una estrategia para trabajar en conjunto tanto de persona a persona como de empresa a empresa, para enfrentar las problemáticas que se presentan $\mathrm{y}$, así, obtener éxito conjunto (Chinomona y Omoruyi, 2016:347). Las cadenas de suministro socialmente responsables tienen como objetivo fundamental 
la optimización de los recursos, el mejoramiento continuo de condiciones de trabajo y el bien común (Yadlapalli et al., 2018:137).

La RSCS ha generado un gran impacto como tema de investigación los últimos años. Feng et al (2017:297) analizan la importancia de las partes interesadas conforme a los enfoques de sostenibilidad y los tipos de análisis implementados para efectuar los cambios que se presentan en cada organización; además, establecen estándares para proveedores e intentan evitar incumplimientos por parte de estos, por medio de un programa de inspección y verificación. Ciliberti et al (2008:1580) establecen que las organizaciones pueden utilizar diferentes estrategias de gestión de RSCS para verificar el cumplimiento de los requisitos y aprovechar la capacidad del proveedor para dar solución a los problemas que se presenten con la RSCS y, así, promover la cultura del proveedor socialmente responsable.

Chinomona y Omoruyi (2016:347) destacan la relación existente entre la RSCS y la innovación y el efecto positivo de ésta en la eficiencia y la efectividad del área de operación comercial, los productos y servicios; también, se destaca la necesidad de cambios en las operaciones en las empresas que son socialmente responsables con sus cadenas de suministro. Por esta razón, las grandes oportunidades de crecimiento de cualquier negocio requieren un comportamiento que sea socialmente responsable al progreso de la comunidad y en la zona en donde se opera (Bisogno, 2016:442).

Sin embargo, algunos autores como Gold y Schleper (2017:428) consideran que aún faltan aportes teóricos sobre la composición de la sostenibilidad en todas las operaciones, productos $y$ procesos en la cadena de suministro. Otros autores como Fernández et al (2014:245) establecen la necesidad de considerar las percepciones de los clientes en las prácticas de RSCS e incorporar los beneficios en los precios de los productos y el factor de RSCS como instrumento de ventaja competitiva.

Se destaca entonces, que la mayoría de aportes teóricos al concepto de RSCS hacen hincapié en los mecanismos de coordinación e integración de estrategias de sostenibilidad para involucrar y generar el compromiso real de todos los miembros de la cadena de suministro. También es fundamental diseñar e implementar objetivos e indicadores de desempeño con un enfoque de sostenibilidad integral que trascienda la perspectiva económica-financiera.

\section{Prácticas de responsabilidad sostenible de cadenas de suministro}

En cuanto a las prácticas de RSCS, se identificaron estudios de revisión crítica de la literatura sobre el estado actual de la sostenibilidad en las cadenas de suministro futuras tanto en la parte académica como en la industrial (Pedram et al, 2017:2), en los cuales se afirma que aunque la sostenibilidad ha tenido repercusión en la cadena de suministro y todas sus operaciones, en algunos casos tiene menos relevancia que otros temas como el desempeño financiero tradicional.

También, se ha estudiado el tema en el contexto de las pequeñas y medianas empresas y sus respectivos comportamientos socialmente respon- 
sables a lo largo de las cadenas de suministro (Ciliberti et al, 2008:1585). Sin embargo, otros autores como Boyd et al, (2007:347) consideran que la implementación de acciones para el mejoramiento en las prácticas de RSCS es independiente del tamaño de las organizaciones. Para llevar a cabo la transición a modelos que sean más sostenibles en cadenas de suministro, se incluyen los códigos, las preocupaciones sociales, así como la colaboración entre las partes interesadas (Quarshie et al, 2016:90).

Otros abordajes de la RSCS, ha sido desde el enfoque de la certificación de proveedores, integrando asuntos sociales para la valoración de prácticas de responsabilidad social, considerando que se generan beneficios a largo plazo y se mejora la eficiencia gerencial en la cadena de suministro (Rahim, 2017:811) y el valor agregado al cliente en el ámbito operacional (KoggyMont, 2012:155). El mejoramiento de las capacidades de los proveedores se refleja directamente en la mejora de las operaciones de producción o prestación del servicio de toda la cadena de suministro (Yawar y Seuring, 2018:233).

Cuando se realizan compras respetuosas con el medio ambiente como parte de las prácticas de RSCS se obtienen ganancias sostenibles, primordialmente desde los aspectos económico y social. Algunos estudios ratifican que las prácticas de RSCS tienen un enlace positivo con el cumplimiento financiero (Hong et al, 2018:3510). En general, han probado empíricamente que estas prácticas pueden lograr efectos positivos de logística inversa y, también, impulsar la competitividad.

El desempeño de coordinación de la cadena de suministro y la productividad de la organización focal extiende una sensación de sostenibilidad significativa a toda la cadena de suministro (Hong et al, 2018:3511). De este modo, las prácticas de RSCS impulsan las actitudes y reacciones de proveedores y clientes y se pueden integrar a los esfuerzos de otros componentes impulsores de ventas como el mercadeo y la publicidad (Wei y Huang, 2017:3).

Los requerimientos de acatamiento ambiental para los proveedores por parte de los compradores y las prácticas de gestión del ciclo de vida del producto tienen beneficios positivos sociales y ambientales (Mariadoss et al, 2016:3409). Por medio de la RSCS se pueden determinar las problemáticas sociales y su conexión con el desempeño global de la cadena de suministro (Mani et al, 2018:261). Sin embargo, la sostenibilidad social en la cadena de suministro se enfrenta a procesos y productos que ocasionan impactos negativos a largo plazo, principalmente, entre las partes interesadas. Por tanto, con el fin de desarrollar una gestión sostenible efectiva, las organizaciones y sus cadenas de suministro requieren integrar requisitos sostenibles en la distinción, valoración y contribución de proveedores (Rueda et al, 2017:2481), considerando aspectos de interés social como el cumplimiento de los derechos humanos y las condiciones de trabajo (Sancha et al, 2016:1936).

Por ejemplo, Shen et al, (2017:2) desarrollaron un modelo para la evaluación y selección de proveedores con base en el Triple Balance; también realizaron un análisis de sensibilidad para la verificación de la evaluación a proveedores verificando la necesidad de tener un enlace de colaboración con los proveedores para así reducir riesgos en ámbitos sociales. Indican, también, que un clima ético puede servir para 
mejorar la actitud en los empleados y el desempeño laboral.

Desde el ámbito de la gestión de la cadena de suministro, Parmigiani et al (2011:220) realizaron una intervención complementando los desempeños sociales y ambientales a las consecuencias económicas definidas por el precio del producto, la flexibilidad, la calidad y la entrega. Demostraron que conservar relaciones firmes con los proveedores es un aspecto relevante y determinante para la integración de aspectos sociales y ambientales. En consecuencia, la gestión simultánea de los proveedores y grupos de interés externos fomenta el fortalecimiento mutuo (Yadlapalli et al,2018:136).

Se requiere un marco de evaluación que identifique el estado real de la implementación de la RSCS en el contexto de organizaciones con cadenas de suministro globales. En ciertos países se realizan aportaciones conformes a la generación de valor del empleo y la generación de valor económico, pero, en otros, es escasa la obtención de información y conocimiento de los flujos de valor en las cadenas de suministro (Baumann et al., 2013:4). También, se requiere incrementar la investigación sobre la agrupación de prácticas de RSCS de corporaciones y el efecto de los incentivos, basados en contratos extensos y gran variedad de pedidos, en el crecimiento y desarrollo de los proveedores (Quarshie et al, 2016:87).

\section{Metodología para la implementación de prácticas de Responsabilidad Sostenible de Cadenas de Suministro}

En el diagrama 3 se muestra la metodología propuesta para la implementación de prácticas de RSCS. Esta propuesta se basa en los resultados mostrados en la sección sobre aportes teóricos y de prácticas de RSCS identificados en la revisión de literatura. Esta metodología sirve de punto de partida para una posterior aplicación del constructo investigado en una cadena de suministro específica.

a. Evaluar partes interesadas pertinentes: la metodología parte de la evaluación de las partes interesadas pertinentes en la cadena de suministro; esto es particularmente importante en cadenas de suministro con un gran número de socios, donde se dificulta la gestión de las prácticas de RSCS en la totalidad de ellos, por lo que se deben identificar y seleccionar las partes interesadas pertinentes para obtener resultados positivos, a través de la aplicación de metodologías cuantitativas adecuadas.

b. Desarrollar estrategias de comunicación y gestión: se deben desarrollar estrategias de comunicación y gestión de RSCS orientadas a la sensibilización y generación de compromiso en todos los miembros de la cadena de suministro seleccionados en la etapa previa.

c. Desarrollar e implementar políticas y prácticas de RSCS: la empresa focal desarrolla e implementa las políticas y prácticas de RSCS a través de mecanismos de cooperación que faciliten su fácil y rápida adopción en un periodo de tiempo establecido y diferencial de acuerdo con las particularidades de cada parte interesada. Se deben desarrollar e implementar objetivos e indicadores de desempeño sostenible comunes y concertados. 
pp. 668-683

\section{Diagrama 3 \\ Metodología propuesta para la implementación de prácticas de RSCS}

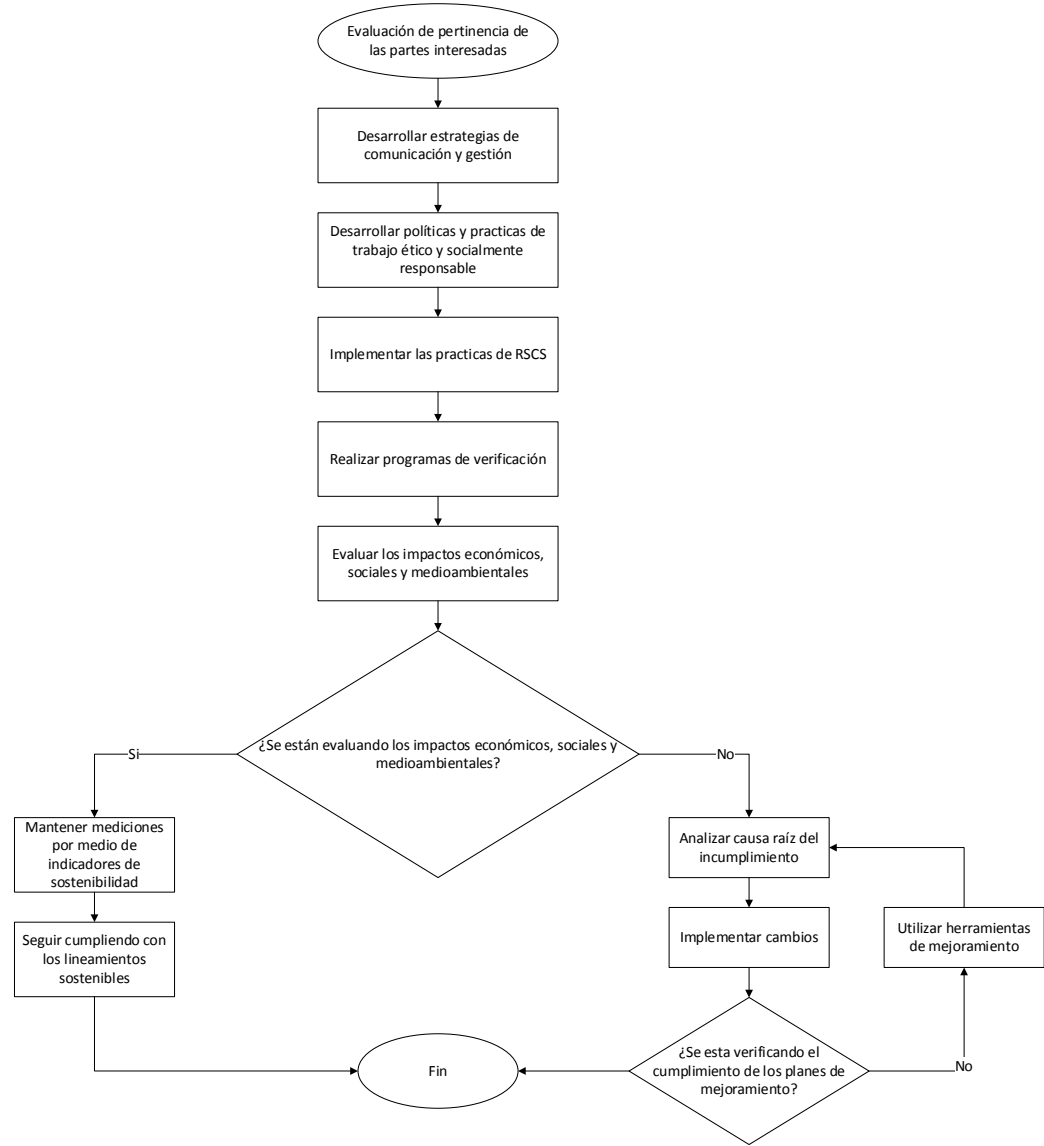

Fuente: Elaboración propia

d. Verificar la implementación: transcurrido el tiempo de implementación, se realiza la verificación de cumplimiento a través de la evaluación de los indicadores económicos, sociales y ambientales previamente concertados. En caso de resultados positivos, se continua con los controles y protocolos ya implementados hasta un nuevo periodo de revisión. En caso de incumplimiento, se debe realizar un análisis de causa raíz e implementar los cambios (corrección y/o acción correctiva) con el fin de garantizar el cumplimiento de los objetivos de sostenibilidad en la siguiente verificación. 
e. Retroalimentación y mejora: los resultados de la evaluación de la fase previa sirven de insumo para el próximo periodo de ejecución, con el fin de eliminar los efectos residuales en los aspectos donde no se haya logrado un desempeño satisfactorio completo y para actualizar las nuevas políticas, objetivos e indicadores que evidencien un proceso de mejora continua desde la visión de la responsabilidad sostenible de la cadena de suministro.

\section{Conclusiones}

La integración de restricciones y objetivos económicos, sociales y ambientales es un aspecto básico en la gestión de las cadenas de suministro modernas y globalizadas. El liderazgo de la cadena de suministro es fundamental para el rendimiento sostenible y para motivar a sus integrantes al afrontar los obstáculos de mayor relevancia que se presentan en la implementación de prácticas de RSCS.

Una cadena de suministro socialmente responsable desarrolla prácticas conjuntas que impactan positivamente en las condiciones de trabajo, la optimización de recursos, el cuidado del medio ambiente y la eficiencia de las operaciones. Para la adopción de estas prácticas, las organizaciones deben implementar estrategias previas de comunicación y coordinación con sus socios de la cadena, con el fin de garantizar su despliegue a lo largo de la cadena de suministro y su permanencia en el mediano y largo plazo.

Se destaca la identificación de la falta de un marco de evaluación general para la implementación de la RSCS en el contexto de cadenas de suministro con operaciones globales, dado que las concepciones de la sostenibilidad y la priorización de los aspectos económico, ambiental y social difiere entre países y culturas. También, se requiere incrementar los desarrollos académicos y prácticos en lo referente al agrupamiento o consolidación de prácticas de RSCS y el efecto de los mecanismos de coordinación e integración en el desarrollo y competitividad de los socios de la cadena de suministro.

Similarmente, la metodología propuesta para la implementación de prácticas de RSCS constituye un punto de partida con bases académicas sólidas y visión integral para futuros trabajos orientados a su implementación y adaptación, dependiendo de las particularidades del sector empresarial y la cadena de suministro seleccionada.

Para finalizar, se destaca que el artículo que se presenta es un resultado inicial de una investigación en curso orientada al desarrollo e implementación de un modelo analítico de prácticas de RSCS usando modelado multiagentes. Las siguientes etapas de la investigación se enfocarán en la especificación de los niveles y agentes del modelo y sus estrategias de coordinación e interacción, asumiéndose como futuras líneas de investigación.

\section{Referencias bibliográficas}

Ağan, Yabuz, Kuzey, Cemil, Acar, Mehmet y Açıkgöz, Atif (2016), The relationships between corporate social responsibility, environmental supplier development, and firm performance. Journal of Cleaner Production, volumen 112, Netherlands, Elsevier, pp.1872-1881. https://doi. org/10.1016/j.jclepro.2014.08.090

Baumann, Dorothée, Wickert, Christopher, Spence, Laura y Scherer, Andreas (2013), Organizing Corporate 
Social Responsibility in Small and Large Firms: Size Matters. Journal of Business Ethics, volumen 115, $\mathrm{N}^{\circ}$ 4, Netherlands, Kluwer Academic Publishers, pp.693-705. https://doi. org/10.1007/s10551-013-1827-7

Benoit, Catherine, Aulisio, Deana y Norris, Gregory (2012), Identifying social impacts in product supply chains: Overview and application of the social hotspot database. Sustainability, volumen $4, \mathrm{~N}^{\circ} 9$, Switzerland, pp.1946-1965. https://doi. org/10.3390/su4091946

Bisogno, Maria (2016), Corporate Social Responsibility and Supply Chains: Contribution to the Sustainability of Well-being. Agriculture and Agricultural Science Procedia, volumen 8, Netherlands, Elsevier, pp.441448. https://doi.org/10.1016/j.aaspro.2016.02.041

Boyd, Eric, Spekman, Robert, Kamauff, John y Werhane, Patricia (2007), Corporate Social Responsibility in Global Supply Chains: A Procedural Justice Perspective. Long Range Planning, volume 40, $\mathrm{N}^{\circ} 3$, United Kingdom, Elsevier, pp.341-356. https://doi. org/10.1016/j.Irp.2006.12.007

Chinomona, Elizabeth, y Omoruyi, Osas (2016), The Influence of Csr, Innovation and Supply Chain Partnership on Firm Competitiveness. Risk Governance and Control: Financial Markets \& Institutions, volumen $6, \mathrm{~N}^{\circ} 4$, Ukraine, Virtus Interpress, pp.345354. https://doi.org/10.22495/rgcv6i4c2art12

Ciliberti, Francesco, Pontrandolfo, Pierpaolo y Scozzi, Barbara (2008), Investigating corporate social responsibility in supply chains: a SME perspective. Journal of Cleaner Production, volumen 16, $\mathrm{N}^{\circ} 15$, Netherlands, Elsevier, pp.15791588. https://doi.org/10.1016/j.jcle-

\section{pro.2008.04.016}

Feng, Yunting, Zhu, Qinghua y Lai, Kee H. (2017), Corporate social responsibility for supply chain management: A literature review and bibliometric analysis. Journal of Cleaner Production, volumen 158, Netherlands, Elsevier, pp.296-307. https://doi. org/10.1016/j.jclepro.2017.05.018

Fernandez, Belen, Romero, Silvia y Ruiz, Silvia (2014), Commitment to Corporate social responsibility measured through global reporting initiative reporting: Factors affecting the behavior of companies. Journal of Cleaner Production, volumen 81, Netherlands, Elsevier, pp.244254. https://doi.org/10.1016/j.jclepro.2014.06.034

Ferrara, Massimiliano, Khademi, Mehrnoosh, Salimi, Mehdi y Sharifi, Somayeh (2017), A Dynamic Stackelberg Game of Supply Chain for a Corporate Social Responsibility. Discrete Dynamics in Nature and Society, volumen 2017 , Egypt, Hindawi, pp. 1-8. https://doi. org/10.1155/2017/8656174

Gold, Stefan y Schleper, Martin (2017), A pathway towards true sustainability: A recognition foundation of sustainable supply chain management. European Management Journal, volumen 35, $\mathrm{N}^{\circ} 4$, United Kingdom, Elsevier, pp.425-429. https://doi. org/10.1016/j.emj.2017.06.008

Hong, Jiangtao, Zhang, Yibin y Ding, Minqiu (2018), Sustainable supply chain management practices, supply chain dynamic capabilities, and enterprise performance. Journal of Cleaner Production, volumen 172, Netherlands, Elsevier, pp.35083519. https://doi.org/10.1016/j.jclepro.2017.06.093

Hutchins, Margot y Sutherland, John (2008), An exploration of measures 
of social sustainability and their application to supply chain decisions. Journal of Cleaner Production, volumen 16, $\mathrm{N}^{\circ} 15$, Netherlands, Elsevier, pp.1688-1698. https://doi. org/10.1016/j.jclepro.2008.06.001

Kitchenham, Barbara (2004), Procedures for Performing Systematic Reviews. Joint Technical Report. Australia: Department of Computer Science. Keele University.

Kogg, Beatrice y Mont, Oksana (2012), Environmental and social responsibility in supply chains: The practise of choice and inter-organisational management. Ecological Economics, volumen 83, Netherlands, Elsevier, pp.154-163. https://doi. org/10.1016/j.ecolecon.2011.08.023

Mani, Venkatesh, Gunasekaran, Angappa y Delgado, Catarina (2018), Enhancing supply chain performance through supplier social sustainability: An emerging economy perspective. International Journal of Production Economics, volumen 195, Netherlands, Elsevier, pp.259-272. https:// doi.org/10.1016/j.jpe.2017.10.025

Mani, Venkatesh, Gunasekaran, Angappa, Papadopoulos, Thanos, Hazen, Benjamin y Dubey, Rameshwar (2016), Supply chain social sustainability for developing nations: Evidence from India. Resources, Conservation and Recycling, volumen 111, Netherlands, Elsevier, pp.4252. https://doi.org/10.1016/j.resconrec.2016.04.003

Mariadoss, Babu, Chi, Ting, Tansujah, Patriya y Pomirleanu, Nadia (2016), Influences of Firm Orientations on Sustainable Supply Chain Management. Journal of Business Research, volumen $69, \mathrm{~N}^{\circ} 9$, Netherlands, Elsevier, pp.3406-3414. https://doi.org/10.1016/j.jbusres.2016.02.003
Parmigiani, Anne, Klassen, Robert y Russo, Michael (2011), Efficiency meets accountability: Performance implications of supply chain configuration, control, and capabilities. Journal of Operations Management, volumen 29, $\mathrm{N}^{\circ} 3$, Netherlands, Elsevier, pp.212-223. https://doi. org/10.1016/j.jom.2011.01.001

Pedram, Ali, Pedram, Payam, Bin Yusoff, Nukman y Sorooshian, Shahryar (2017), Development of closedloop supply chain network in terms of corporate social responsibility. PLoS One, volumen 12, $\mathrm{N}^{\circ} 4$, United States, pp.1-20. https://doi. org/10.1371/journal.pone.0174951

Plasencia, Antonio, Marrero, Fernando, Bajo, Anna y Nicado, Miriam (2018), Modelos para evaluar la sostenibilidad de las organizaciones. EG Estudios Gerenciales, volumen 34, N¹46, Colombia, Universidad ICESI, pp.63-73. https://doi.org/10.18046/j. estger.2018.146.2662

Quarshie, Anne, Salmi, Asta y Leuschner, Rudolf (2016), Sustainability and corporate social responsibility in supply chains: The state of research in supply chain management and business ethics journals. Journal of Purchasing and Supply Management, volumen $22, \mathrm{~N}^{\circ} 2$, United Kingdom, Elsevier, pp.82-97. https://doi. org/10.1016/j.pursup.2015.11.001

Rahim, Mia (2017), Improving Social Responsibility in RMG Industries Through a New Governance Approach in Laws. Journal of Business Ethics, volumen 143, $\mathrm{N}^{\circ} 4$, Netherlands, pp.807-826. https://doi. org/10.1007/s10551-016-3131-9

Rueda, Ximena, Garrett, Rachael y Lambin, Eric (2017), Corporate investments in supply chain sustainability: Selecting instruments in the agri-food industry. Journal of 
Cleaner Production, volume 142, Netherlands, Elsevier, pp.24802492. https://doi.org/10.1016/j.jclepro.2016.11.026

Sancha, Cristina, Gimenez, Cristina y Sierra, Vicenta (2016), Achieving a socially responsible supply chain through assessment and collaboration. Journal of Cleaner Production, volumen 112, Netherlands, Elsevier, pp.1934-1947. https://doi. org/10.1016/j.jclepro.2015.04.137

Shen, Bin, Li, Qingying, Dong, Ciwei y Perry, Patsy (2017), Sustainability Issues in Textile and Apparel Supply Chains. Sustainability, volumen 9, $\mathrm{N}^{\circ}$ 9, Switzerland, MDPI, pp.1-6. https://doi.org/10.3390/su9091592

Sudarto, Sumarsono, Takahashi, Katsuhiko y Morikawa, Katsumi (2017), Efficient flexible long-term capacity planning for optimal sustainability dimensions performance of reverse logistics social responsibility: A system dynamics approach. International Journal of Production Economics, volumen 184, Netherlands, Elsevier, pp.179-192. https://doi. org/10.1016/j.jpe.2016.12.013

Wang, Baoying (2013), Research on cor- porate social responsibility of supply chain system based on the self-organization theory. Research Journal of Applied Sciences, Engineering and Technology, volumen $5, \mathrm{~N}^{\circ} 10$, United Kingdom, pp.2985-2990. https:// doi.org/10.19026/rjaset.5.4611

Wei, Yu y Huang, Shih (2017), Food traceability system as elevating good corporate social responsibility for fast-food restaurants. Cogent Business \& Management, volumen 4, $\mathrm{N}^{\circ}$ 1, Cogent, pp.1-10. https://doi.org/10 $.1080 / 23311975.2017 .1290891$

Yadlapalli, Aswini, Rahman, Shams y Gunasekaran, Angappa (2018), Socially responsible governance mechanisms for manufacturing firms in apparel supply chains. International Journal of Production Economics, volumen 196, Netherlands, Elsevier, pp.135-149. https://doi. org/10.1016/j.ijpe.2017.11.016

Yawar, Sadaat y Seuring, Stefan (2018), The Role of Supplier Development in Managing Social and Societal Issues in Supply Chains. Journal of Cleaner Production, volumen 182, Netherlands, Elsevier, pp.227237. https://doi.org/10.1016/j.jclepro.2018.01.234

- $\quad$ Esta obra está bajo una licencia de Creative Commons Reconocimiento-NoComercial- Compartirlgual 3.0 Unported. http://creativecommons.org/licenses/by-nc-sa/3.0/deed.es_ES 